\title{
Surgical Management of Renal Hyperparathyroidism: Case Series and Review of the Literature
} \author{
Angela $^{5}$, Sala Daniela ${ }^{1}$ \\ 1 Second Department of General Surgery, University of Medicine and Pharmacy Tîrgu Mureș, Romania \\ 2 Centrul de Dializă B Braun Avitum Tîrgu Mures \\ ${ }^{3}$ Nephrology Department, University of Medicine and Pharmacy Târgu Mureș, Romania \\ 4 Endocrinology Department, University of Medicine and Pharmacy Târgu Mureș, Romania \\ 5 Department of Histology, University of Medicine and Pharmacy Târgu Mureș, Romania
}

Neagoe Radu Mircea¹, Mureșan Mircea1* ${ }^{\star}$, Văcar Smaranda², Hossu loan³, Pașcanu lonela4, Borda

\begin{abstract}
Secondary hyperparathyroidism (SHPT) occurs most commonly in the setting of chronic renal failure (CRF) being frequently referred to as "renal" hyperparathyroidism The "classical" medical treatment with oral calcium and vitamin D supplementation is generally sufficient to lower parathyroid hormone levels in the majority of these patients. However, we frequently encounter cases of severe refractory sHPT, a state in which even recently available therapeutic agents, i.e. calcimimetics, new phosphate binders, vitamin D analogues, remain inefficient, thus parathyroidectomy and/ or renal transplant becoming necessary. Three types of surgeries have been proposed in sHPT: two of them are grouped as remnant-conserving techniques, i.e. subtotal

parathyroidectomy (sPtx) and total parathyroidectomy with autotransplantation ( $\mathrm{PPtx}+\mathrm{AT}$ ), the third one being total parathyroidectomy without autotransplantation (tPtx). There was a continuous debate concerning the best surgical approach in renal hyperparathyroidism, starting very soon after those techniques were described; without pretending to solve these controversies, this paper aims to review the surgical treatment options in sHPT, based on our 5-year experience in dealing with the disease.
\end{abstract}

Keywords: renal hyperparathyroidism, subtotal parathyroidectomy, total parathyroidectomy with autotransplant

Received: 15 June 2015 / Accepted: 04 August 2015

\section{Introduction}

Secondary hyperparathyroidism (sHPT) occurs most commonly in the setting of chronic renal failure (CRF) being frequently referred to as "renal" hyperparathyroidism. The disease appears almost as a rule in the final stages of CRF, approximately $90 \%$ of CRF patients developing $\mathrm{sHPT}$ by the time hemodialysis becomes necessary; other less frequent causes of sHPT include malabsorbtion and vitamin D deficiency $[1,2]$. The "classical" medical treatment with oral calcium and vitamin D supplementation is generally sufficient to lower parathyroid hormone levels in the majority of these patients [3]. However, we frequently encounter cases of severe refractory sHPT, a state in which even recently available therapeutic agents, i.e. calcimimetics, new phosphate binders, vitamin $\mathrm{D}$ analogues, remain inefficient, thus parathyroidectomy and/ or renal transplant becoming necessary [4].

Three types of surgeries have been described in the past 50 years for the surgical management of "renal" hyperparathyroidism, each of them having their proponents and opponents. Remnant-conserving techniques, which leave a small portion of parathyroid tissue either in the neck or at distance, were incriminated with having high recurrent disease rates while the new "rehabilitated" total parathyroidectomy without autotransplantation seems to deter-

* Correspondence to: Mircea Muresan

E-mail: dr_muremir@yahoo.com mine a long-standing hypoparathyroid status which finally affects bone metabolism $[5,6]$.

Without pretending to solve these controversies, this paper aims to review the surgical treatment options in sHPT, based on our 5-year experience in dealing with the disease.

\section{Indications for surgery in renal hyperparathyroidism and pre- operative work-up}

The medical treatment of sHPT enriched in the last 10-15 years with new therapeutic agents such as calcimimetics, vitamin $\mathrm{D}$ analogues or new phosphate binders, all designed to manage refractory cases and bridge patients to the optimal treatment, i.e. renal transplant [2]. However, between 1-2 \% of patients with sHPT still need parathyroidectomy each year [7].

Among the surgical criteria for parathyroidectomy the following are largely accepted $[2,3,5,8]$ :

- severe medically refractory sHPT, with intact parathyroid hormone (iPTH) seric values constantly above $700 \mathrm{pg} / \mathrm{ml}$ (nv:15-67pg/ml), hyperphosphatemia and hypercalcemia (calcium phosphorus product Ca x P > 70)

- severe osteoporosis and clinical symptoms: pruritus, bone pains, soft tissue and vascular calcification, history of renal calculi or pathologic bone fractures, brown tumors and other aspects of osteitis fibrosa cystica 
- calciphylaxis

- enlarged parathyroid glands, with at least one gland over $0.5 \mathrm{~cm}^{3}$ in volume or $1 \mathrm{~cm}$ in diameter on ultrasonography

- medical observation not possible

Apart from the clinical and laboratory criteria partially mentioned above, the pre-operative work-up in sHPT also includes imagistic localization studies. Even though the sensitivity of these imaging studies is low in sHPT when compared with primary hyperparathyroidism [2] there are authors who recommend both ultrasonography and 99mTc-sestamibi in either initial diagnosis of sHPT or in reinterventions [14]. In recent years, other imagistic techniques have been described in sHPT diagnosis such as computed tomography (CT), magnetic resonance imaging (MRI), single photon-emission tomography (SPECT); however, the studies regarding these investigations include too few patients to allow pertinent conclusions [2]. Like other researchers $[2,5,8]$, we do not consider localization studies as mandatory in the initial diagnosis of sHPT for at least two reasons: firstly, imaging examinations fail to "locate" all parathyroids, including ectopies, in patients with multiple gland disease, which is the case in sHPT, and, secondly, the surgical approach in sHPT is unlikely to change, i.e. bilateral neck exploration, regardless of what localization studies describe (Figure 1A,B).

\section{Surgical approaches in renal hyperparathyroidism}

Three types of surgeries have been proposed in sHPT: two of them are grouped as remnant-conserving techniques, i.e. subtotal parathyroidectomy ( $\mathrm{P}$ tx $)$ and total parathyroidectomy with autotransplantation $(\mathrm{tPtx}+\mathrm{AT})$, the third one being total parathyroidectomy without autotransplantation (tPtx). There was a continuous debate concerning the best surgical approach in renal hyperparathyroidism, starting very soon after those techniques were described; finally it seems that surgeon preference remains the main argument in this decision making process $[6,11]$.

Subtotal parathyroidectomy was first proposed by Stanbury in the 1960s, and consists of removing all but a portion of one gland, this "remnant" being left in situ [12]. The procedure was generally embraced for more than a decade, with good short- and medium-term results reported, being considered at that time the standard of surgical care for renal hyperparathyroidism [13]. The initial enthusiasm was soon overshadowed by the higher rates of persistent and recurrent diseases observed on long-term follow-up; furthermore the reoperation in the neck for relapsing disease comes with technical challenges in avoiding damages of important anatomic structures, i.e. recurrent laryngeal nerves $[6,11,14]$. It was because all of these drawbacks that another surgical technique was later popularized, namely, total parathyroidectomy with autotransplantation of parathyroid tissue. In 1975 Wells described the well-known surgical procedure which bears his name - total parathyroidectomy with autotransplantation in anterior forearm musculature [15]. Wells' technique has some important advantages when compared with sPtx, mainly as regards either the localization of recurrent hyperplasic parathyroid tissue in the forearm through 99 Tc-sestamibi scintigraphy or reoperation in the latter region. Several retrospective analyses and one randomized trial compared Wells' technique with sPtx, with puzzling conclusions; currently there is a general consensus that the results of both procedures are comparable $[11,16]$. However, Rothmund et al. rand-
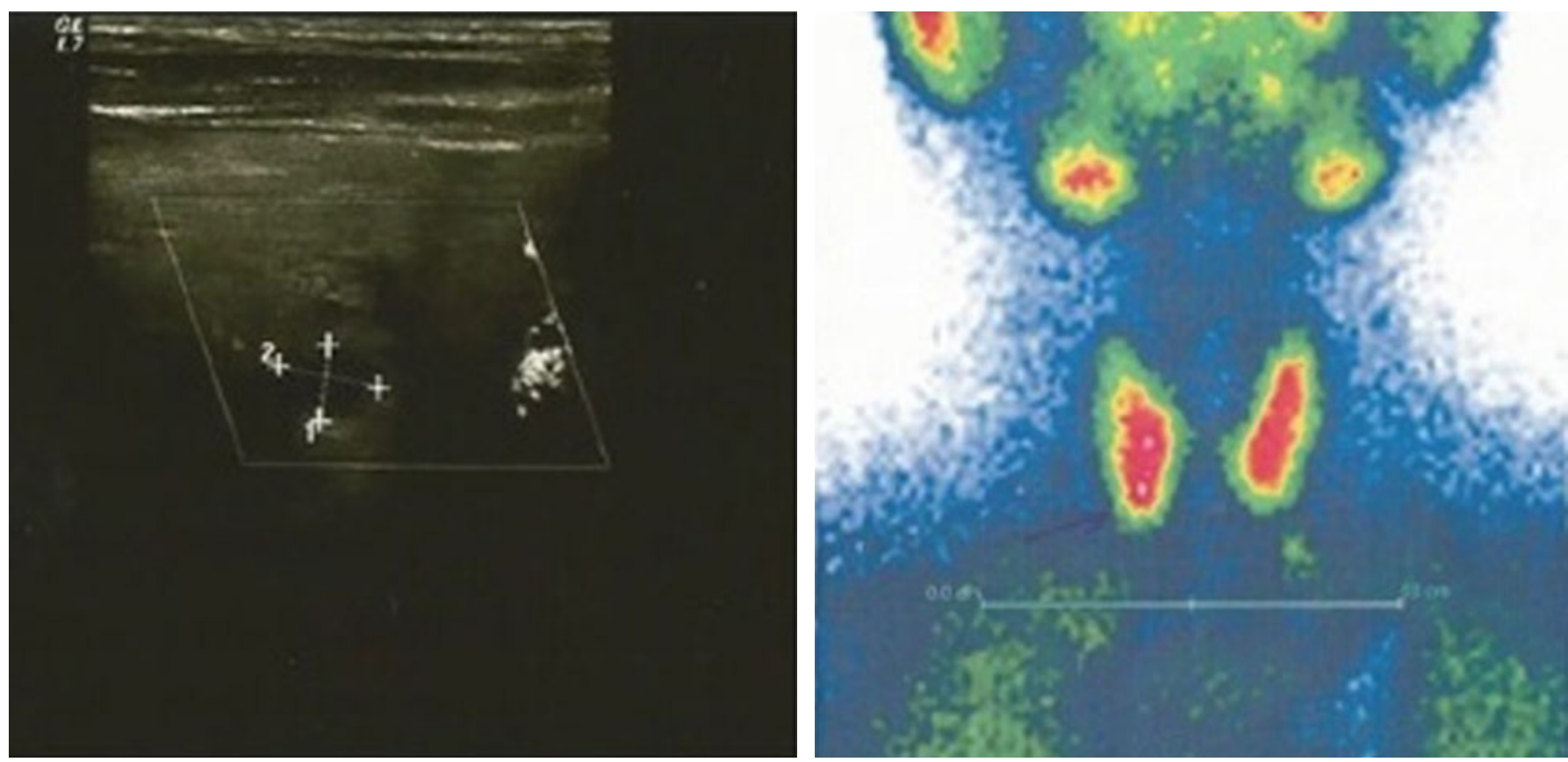

Fig. 1. Localization studies in a case of renal hyperparathyroidism. A-cervical ultrasonography showing an hypoechoic nodule located at the inferior part of the right thyroid lobe, consistent with a right inferior parathyroid adenoma. B- 99mTc-sestamibi confirming the right inferior parathyroid adenoma. 
omized 40 patients to either sPtx or tPtx+AT and described better results after the latter technique as regards improving of symptoms, cure rate, and recurrent disease [17].

We perform sPtx with a technical particularity regarding the position of the remnant tissue in the neck; we do not abandon parathyroid tissue in situ as described by the majority of the authors $[5,8,13]$, including the original paper [12], but instead we choose an inferior parathyroid gland, "lift" it in a suprasternal position, above the strap muscles. We then resect the gland subtotally, conserving a less modified portion with its caudal vascularization through a "neopedicle" represented by small mediastinal and thymic vessels (Figure 2AB). Similarly to other authors [18] we consider that this technique has both an "anatomical" advantage of an easy operative access in case of recurrent hyperparathyroidism and also a "physiological" one, concerning the more rapid function of the vascularized autotransplanted parathyroid tissue. We started performing sPtx accordingly in February 2010, our initial, short- and medium-term results of the first 24 cases having been previously reported $[19,20]$. This initial cohort included 16 women $(66.7 \%)$ and 8 men, with a mean age of $50 \pm 10.6$ years, ranging between 31 and 69 years; what characterized our lot was the long evolution of the disease, the average time of hemodialysis being 8.35 years. All patients had important disease-related symptoms i.e. osteoarticular pains and bone changes, pathological fractures, pruritus, soft tissue calcification; we also encountered a very rare case of multiple spine brown tumors in a female patient with a preoperative iPTH of $10000 \mathrm{pg} / \mathrm{ml}$. In fact all our patients had markedly elevated preoperative iPTH levels, with a median value of $2131 \mathrm{pg} / \mathrm{ml}$, along with hyperphosphataemia and high levels of alkaline phosphatase. All symptoms and biochemical parameters markedly improved after sPtx; immediate postoperative iPth level decreased by 150.1 times, the first month median value being $28 \mathrm{pg} / \mathrm{ml}$, these values remaining significantly decreased in the follow-up period

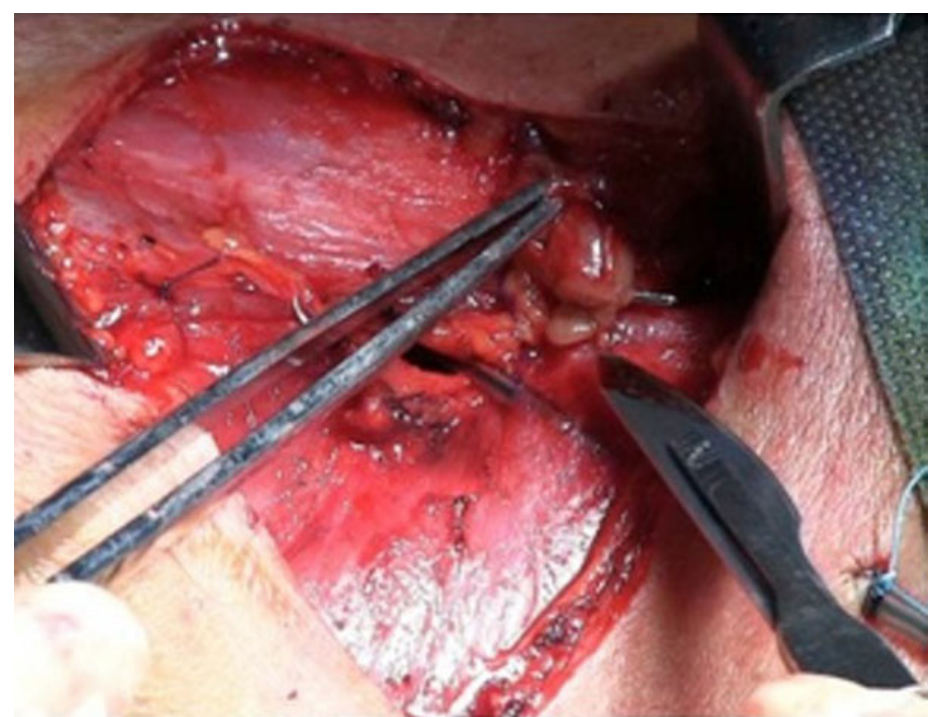

of 6-18 months. Furthermore, we noted a normalization of serum calcium, phosphorus and alkaline phosphatase in the majority of patients in the aforementioned follow-up period.

Regarding $\mathrm{tPtx}+\mathrm{AT}$ we favored the sternocleidomastoid muscle as the receptor for the remnant tissue, due to the simplicity and rapidity of the technique. After the total resection of the parathyroids from the neck we choose the less structural modified gland, cut 10-12 small pieces of $1 \mathrm{~mm}$, further implanting them in 2-3 muscular pockets. Using the sternocleiodomastoid muscle as receptor for transplantation was intensely criticized by many authors, arguing the difficulties in localization of the recurrent hyperplasic tissue in the neck, esthetic and functional reasons $[3,11]$. We constantly mark the muscular pockets with a metallic clip, thus facilitating both the localization and the resection of the recurrent hyperplasic parathyroid tissue. Until now 22 patients underwent $\mathrm{Ptx}+\mathrm{AT}$ the partial results of the first 19 cases being comparatively analyzed with the previously mentioned sPtx group (under publication). We concluded that both techniques have comparable results as concerning the clinical and laboratory outcome and rates of postoperative hypoparathyroidism at least in short and medium-term follow-up (Figure $3 \mathrm{~A}, \mathrm{~B}, \mathrm{C}$ ).

Regardless of the chosen approach, a thorough understanding of the anatomy and embryology of the parathyroid glands is deemed necessary, to avoid leaving "unfound" parathyroid tissue in the neck, find "missing" and supernumerary glands and to protect important anatomical structures during dissection, i.e. recurrent laryngeal nerves.

The role of simultaneous thymectomy with either subtotal or total parathyroidectomy is also under debate. Some authors routinely resect the cervical part of the thymus bilaterally, claiming its role in avoiding persistent hyperparathyroidism and recurrent disease [11]. Pattou et al. [21] showed that supernumerary parathyroid glands are found in $30 \%$ of patients with sHPT, being located mainly in the

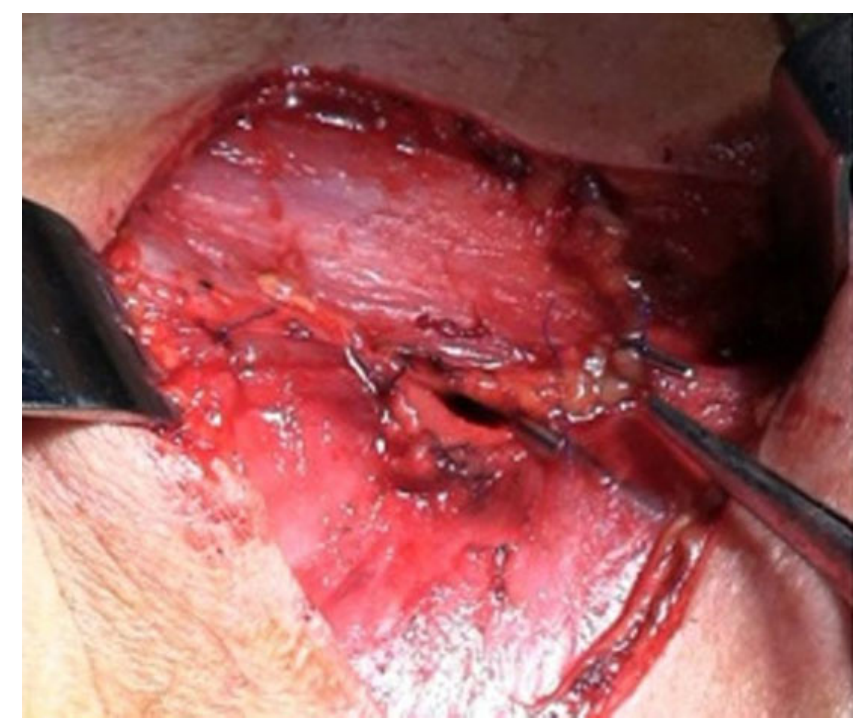

Fig. 2. Subtotal parathyroidectomy;. A- choosen parathyroid gland lifted above strap muscles. B- the parathyroid gland is resected subtotally. 


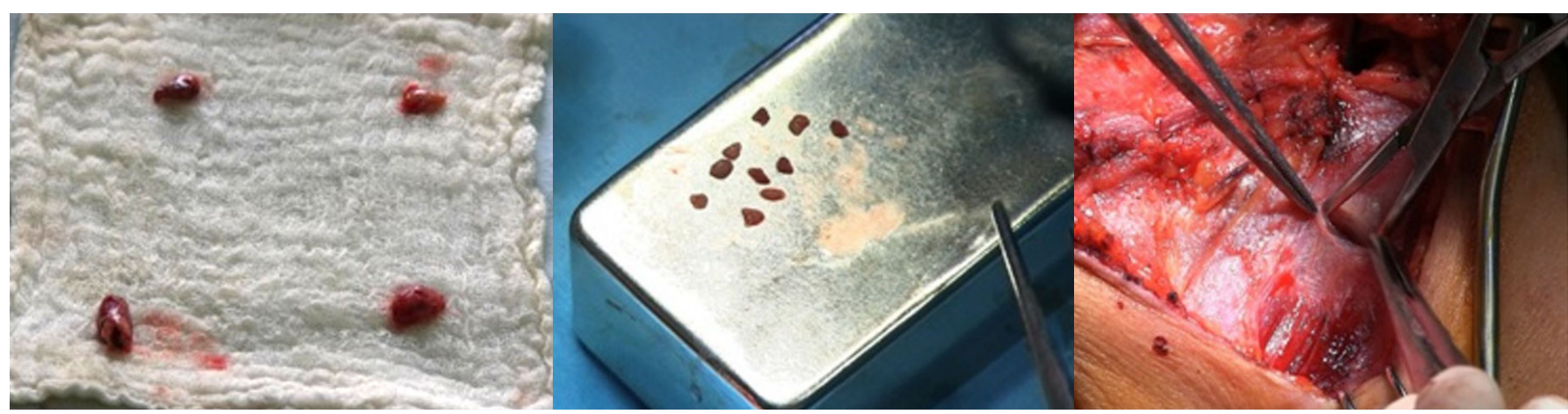

Fig. 3. Total parathyroidectomy with autotransplant in sternocleidomastoid muscle. A- total parathyroidectomy; all (4) parathyroids are resected. B- 10 small pieces of less modified gland prepared for autotransplant. C-parathyroid autotransplant in right sternocleidomastoid muscle

thymus. In opposition, others indicate thymectomy only when less than four glands are found [22], an inferior parathyroid is missing or the radioguided probe indicates an intense radioactivity in the cervical thymus [2]. A similar dilemma concerns the role of routine central compartment lymphadenectomy in the surgical management of renal hyperparathyroidism. As others $[2,3,5]$ we consider it an overdimensioned surgical step, with questionable benefits and possible related complications.

Recently, we noted the advent of a third surgical approach - total parathyroidectomy without autotransplantation ( $\mathrm{tPtx}$ ), with promising results mainly as regards recurrent disease $[6,14,23,24]$. The technique was first performed by Fergusson and reported by Ogg in 1967 [25] but was soon criticized for inducing a severe postoperative hypoparathyroidism and an adynamic bone disease, a state in which low postoperative iPth values determine metabolic and structural impairment of the bones. Due to those purported disadvantages $\mathrm{tPtx}$ was viewed in disfavor for the next two decades, the first encouraging results being reported only after 1990 [26-28]. The major drawback of sPtx and tPtx+AT, i.e. recurrent disease, was in fact the main driving argument for tPtx "rehabilitation". Indeed, many studies reported good short-term results after both remnant- conserving techniques but the disease relapse rates few years afterwards were high $[1,8,13,14,16,17]$. Thus, the 1994 European Dialysis and Transplant Association (EDTA) yearly report noted that $12.8 \%$ of either sPtx or tPtx+AT were performed for recurrent disease [29]. Even though the reported series were not large, the medium- and long-term results after tPtx appear to be good, especially as recurrent disease is concerned. To the best of our knowledge, Puccini et al. [6] reported a series of 43 patients with tPtx with the longest follow-up period of 8.8 years. The authors concluded that the procedure is safe and effective, with low rates of relapsing disease; in spite of higher percentage of hypoparathyroidism, hypocalcemia was easily managed with calcium and vitamin D. Based on our experience, we also consider tPtx a solution for severe sHPT, especially in patients with long-lasting disease and low perspectives of renal transplant. Even if we noted a clear tendency to postoperative hypoparathyroidism in this group of patients, with iPth levels significantly lower than in the other groups (sPtx and tPtx+AT), these patients did not exhibit significantly more severe postoperative hypocalcemia episodes than their counterparts. Furthermore, hypocalcemia episodes were simply treated with calcium supplements and vitamin D.

\section{Conclusion}

Even though the medical treatment of "renal" hyperparathyroidism enriched with effective new therapeutic agents such as calcimimetics and vitamin $\mathrm{D}$ analogues, parathyroidectomy remains a necessity especially in regions where those treatments are still expensive and where there is a clear shortage of organs for renal transplant. All three aforementioned surgical techniques have more or less comparable results, thus the optimal surgical solution remains controversial; the lack of large series, randomized trials, and metaanalyses augment the dilemma, making the analysis more difficult. Finally, surgeon preference and experience remain the main driving arguments in choosing the best technical approach to sHPT. Whichever this approach might be, a bilateral exploration of the neck remains mandatory in the surgical treatment of renal hyperparathyroidism.

\section{Acknowledgment}

This work was supported under the frame of Scientific Research Project no. 14439/15.10.2014 (University of Medicine and Pharmacy Tg. Mures).

\section{References}

1. Memmos D, Williams G, Eastwood J, et al. The role of parathyroidectomy in the management of hyperparathyroidism in patients on maintenance haemodialysis and after renal transplantation. Nephron 1982;30(2):143148.

2. Pitt SC, Sippel RS, Chen H. Secondary and tertiary hyperparathyroidism, state of the art surgical management. Surg Clin North Am. 2009;89(5):1227-39.

3. Madorin C, Owen RP, Fraser WD, et al. The surgical management of renal hyperparathyroidism. Eur Arch Otorhinolaryngol. 2012;269(6):1565-76.

4. Cohen EP, Moulder JE. Parathyroidectomy in chronic renal failure: has medical care reduce the need for surgery? Nephron 2001; 89(3):271274

5. Tominaga Y, Matsuoka S, Uno N, Sato T. Parathyroidectomy for secondary hyperparathyroidism in the era of calcimimetics. Ther Apher Dial. 2008;12 Suppl 1:S21-6.

6. Puccini M, Carpi A, Cupisti A, Caprioli R, lacconi P, Basotti M et al. 
Total parathyroidectomy without autotransplantation for the treatment of secondary hyperparathyroidism associated with chronic kidney disease: clinical and laboratory long-term follow-up. Biomed \& Pharmacother 2010;64:359-362

7. Triponez F, Clark O, Vanrenthergem $\mathrm{Y}$, et al. Surgical treatment of persistent hyperparathyroidism after renal transplantation. Ann Surg 2008;248(1):18-30.

8. Neyer $\mathrm{U}$, Horandner $\mathrm{H}$. Parathyroidectomy in renal hyperparathyroidism CIN 2003. Available at http:// www.uninet.edu/cin2003/conf/neyer/ neyer.html

9. Haciyanli M, Lal G, Morita E, et al. Accuracy of preoperative localization studies and intraoperativeparathyroid hormone assay in patients with primary hyperparathyroidism and double adenoma. J Am Coll Surg 2003;197(5):739-746.

10. Sebag F, Hubbard J, Maweja S, et al. Negative preoperative localization studies are highly predictive of multiglandular disease in sporadic primary hyperparathyroidism. Surgery 2003;134(6):1038-1041.

11. Sakman G, Parsak GK, Balal M, etal. Outcomes of total parathyroidectomy with autotransplantation versus subtotal parathyroidectomy with routine addition of thymectomy to both groups: single center experience of secondary hyperparathyroidism. Balkan Med J. 2014;31(1):77-82.

12. Stanbury SW, Lumb GA, Nicholson WF. Elective subtotal parathyroidectomy for renal hyperparathyroidism. Lancet 1960;1(7128):793-799

13. O Leary DP, White HJO. Parathyroidectomy for hyperparathyroidism associated with renal disease. Ann R Coll Surg Engl 1995; 77(2):97-101

14. Drakopoulus S, Koukulaki M, Apostolou T, et al. Total parathyroidectomy whitout autotransplantation in dialysis patients and renal transplant recipients, long term follow-up evaluation. Am J Surg 2009;198(2):178183.

15. Wells SA, Gunnels CJ, Shelburne JD, Schneider AB, Sherwood LM. Transplantation of the parathyroid gland in a man. Surgery 1975;78(1):34-44.

16. Gagné E, Ureña P, Leite-Silva S, et al. Short- and long-term efficacy of total parathyroidectomy with immediate autografting compared with subtotal parathyroidectomy in hemodialysis patients. J Am Soc Nephrol 1992;3:1008.

17. Rothmund M, Wagner PK, Schark C. Subtotal parathyroidectomy versus total parathyroidectomy and autotransplantation in secondary hyperparathyroidism: a randomized trial. Word J Surg 1991;15:745-50.
18. Perez-Ruiz L, A Betriu, Pelayoe A, Fernandez E. New technique of parathyroidectomy to prevent parathyromatosis and hypoparathyroidism Nephrol D Transplant 1999; 14(6):1553-1555

19. Neagoe RM, Sala DT, Roman V, Voidazan S, Pascanu I. Subtotal parathyroidectomy in the treatment of renal hyperparathyroidism- single center initial experience. Acta Endo(Buc) 2013;9(3):385-95.

20. Neagoe RM, DT Sala, I Pascanu, S Voidazan, R Moldovanu. Parathyroidectomy in the treatment of secondary hyperparathyroidism. Clinical and laboratory outcomes. Rom J Lab Med. 2014;22(3):355-66.

21. Pattou FN, Pellissier LC, Noel C, et al. Supernumerary parathyroid glands: frequency and surgical significance in treatment of renal hyperparathyroidism. World J Surg 2000;24(11):1330-1334.

22. He Q, Zhuang D, Zheng L, et al. Total parathyroidectomy with trace amounts of parathyroid tissue autotransplantation as the treatment of choice for secondary hyperparathyroidism: a single-center experience. BMC Surgery. 2014; 14:26.

23. Shih ML, Duh QY, Hsieh CB, et al. Total parathyroidectomy without autotransplantation for secondary hyperparathyroidism. World J Surg 2009;33(2):248-254.

24. Coulston JE, Egan R, Willis E, Morgan JD. Total parathyroidectomy without autotransplantation for renal hyperparathyroidism. Br J Surg. 2010; 97(11): 1674-1679.

25. Ogg CS. Total parathyroidectomy in treatment of secondary (renal) hyperparathyroidism Br Med J. 1967;4(5575):331-334.

26. Higgins RM, Richardson AJ, Radcliffe PJ, et al. Total parathyroidectomy alone or with autograft for renal hyperparathyroidism? OJ Med 1991;79(288):323-332.

27. Hampl H, Steinmuller T, Frohling $P$, et al. Long-term results of tota parathyroidectomy without autotransplantation in patients with or without renal failure. Miner Electrolyte Metab 1999;25(3):161-170.

28. Ockert S, Willeke F, Richter A, et al. Total parathyroidectomy without autotransplantation as a standard procedure in the treatment of secondary hyperparathyroidism. Langenbecks Arch Surg 2002;387:204-209.

29. Valderrabano F, Berthoux FC, Jones EH, et al. Report on management of renal failure in Europe. XXV end stage renal disease and dialysis report. The 1994 EDTA-ERA Registry. European Dialysis and Transplant Association-European renal association. Nephrol Dial transplant 1996;119(Suppl 1):2-21. 